\title{
Trayectoria normativa de la inteligencia artificial en los países de Latinoamérica con un marco jurídico para la protección de datos: límites y posibilidades de las políticas integradoras
}

Revista Latinoamericana de Economía y Sociedad Digital

Issue 2, agosto 2021

Autores: Alexandre Veronese(D), Amanda Nunes Lopes Espiñeira Lemos(iD)

DOI: $10.53857 / \mathrm{MZBU} 2371$

Publicado: 25 agosto, 2021

Recibido: 23 agosto, 2021

Cita sugerida: Veronese, Alexandre \& Nunes Lopes Espiñeira Lemos, Amanda (2021)

"Trayectoria normativa de la inteligencia artificial en los países de Latinoamérica con un marco jurídico para la protección de datos: límites y posibilidades de las políticas integradoras", en Revista Latinoamericana de Economía y Sociedad Digital, Issue 2

Licencia: Creative Commons Atribución-NoComercial 4.0 Internacional (CC BY-NC 4.0)

Tipo: Análisis de política pública

Palabras clave: inteligencia artificial, Protección de datos

\section{Resumen}

Muchos países de Latinoamérica están invirtiendo en planes o estrategias de inteligencia artificial (IA) y focalizan en la mejora del desarrollo económico. Una de las características fundamentales de las políticas públicas es el marco regulatorio. Además de las ya ampliamente mencionadas guías éticas, se pueden explorar otras trayectorias normativas como la legislación para la protección de los datos personales y los incentivos económicos. El artículo describe las políticas públicas para la IA de los países de la región que tienen sistemas jurídicos de protección de datos. Utiliza un método cualitativo para evaluar las políticas y estrategias de IA en Argentina, Uruguay, Colombia, México, Chile y Brasil, mediante la descripción de las posibilidades regulatorias en lo relacionado con el uso de la protección de datos. Luego de esta descripción, este estudio abre un debate acerca de los límites y problemas con la utilización de la protección de datos para regular a la IA. 
Concluye que los países no ofrecen muchas alternativas para evaluar a las inversiones tecnológicas como una posibilidad normativa, aun cuando todos los otros medios confíen en ellos para ser efectivos. También, establece que estas inversiones deben tener en cuenta a la dimensión del valor social para beneficiar realmente a los países.

\section{Abstract}

Many Latin American countries are investing on artificial intelligence (AI) plans or strategies focusing on improving their economic development. One crucial feature of any of those public policies is the regulatory framework. Besides the widespread mention of ethical guidelines, one can explore other regulatory paths, like personal data protection law, and economic incentives. The paper describes the AI public policies of the region's countries that have data protection legal systems. It uses a qualitative methodology to assess AI policies and strategies in Argentina, Uruguay, Colombia, Mexico, Chile, and Brazil, describing their regulatory possibilities regarding data protection usage. After this description, the paper presents a debate about the limits and problems with using data protection to regulate AI. It concludes that the countries gave little space to assess technological investments as a regulatory possibility, even though all the other means rely on them to become effective. Also, it states that these investments must incorporate some social value dimension to actually benefit the countries.

\section{Resumo}

Muitos países latino-americanos estão investindo em planos ou estratégias de inteligência artificial (IA) com foco na otimização do seu desenvolvimento econômico. Uma característica crucial de qualquer uma dessas políticas públicas é o marco regulatório. Além da menção generalizada de diretrizes éticas, é possível explorar outros caminhos regulatórios, como a lei de proteção de dados pessoais e incentivos econômicos. Este artigo descreve as políticas públicas de IA dos países da região que possuem sistemas jurídicos de proteção de dados. Ele usa uma metodologia qualitativa para avaliar as políticas e estratégias de IA na Argentina, Uruguai, Colômbia, México, Chile e Brasil, descrevendo suas possibilidades regulatórias em relação ao uso de proteção de dados. Após essa descrição, 0 artigo apresenta um debate sobre os limites e problemas do uso de proteção de dados para regular a IA. Conclui que os países deram pouco espaço para avaliar os investimentos tecnológicos como uma possibilidade regulatória, embora todos os demais meios dependam deles para se tornarem efetivos. Além disso, afirma que esses investimentos devem incorporar alguma dimensão de valor social para de fato beneficiar os países. 


\section{Introducción}

La inteligencia artificial (IA) se ha convertido en una materia de gran interés para mejorar las economías a nivel mundial. También demuestra ser decisiva para el futuro de mejores servicios públicos. Las aplicaciones de IA se diseñan para completar vacíos en áreas con procesos engorrosos, como los registros o las demandas judiciales reiterativas. A pesar de los beneficios, los elementos éticos y los temas relativos a la transparencia en estos procesos son dos aspectos preocupantes de estas innovaciones. Este estudio describe el escenario actual de las políticas públicas que promueven a la IA en Latinoamérica y debate sobre los posibles medios regulatorios, en especial la ley de protección de datos. Muchas de las iniciativas de la IA se están convirtiendo en planes nacionales, políticas o estrategias en varios países, ya sea descritos como completos o considerados en progreso. Además, muchas fuentes están haciendo un gran esfuerzo para crear una red de cooperación más amplia a un nivel regional. Como ejemplo podemos mencionar a la Comisión Económica para Latinoamérica y el Caribe (CEPAL) de las Naciones Unidas, que está tratando de proponer ese movimiento. Otro ejemplo proviene de la propuesta de la IA para mejorar ciertos trabajos específicos, como la lucha contra la corrupción, mostrado por la Organización de los Estados Americanos (OEA) (Moss, 2019). La OEA también patrocina a la "Red de gobierno electrónico de América Latina y el Caribe" (RedGEALC), que es una red involucrada de lleno en el avance de esta agenda en la región (RedGEALC, 2021). Además, existen algunas iniciativas no gubernamentales, como IA Latam, que es una red de empresas e investigadores (IA Latam, 2021). Aun el Banco Interamericano de Desarrollo (BDIA) patrocinó un informe entregado por Fair LAC que cubre ampliamente el estado de las políticas de doce países de la región Gómez (Mont et al, 2020). Este estudio se focaliza en el debate de un marco regulatorio posible para algunos países de Latinoamérica y no en la eficacia de esas políticas públicas. Todos los países en estudio tienen estatutos sobre la protección de datos. En relación con la protección de datos en la región, es clave mencionar a la Red Iberoamericana de Protección de Datos Personales y sus constantes esfuerzos para mejorar los estatutos nacionales en este tema (REDIPD, 2021).

Ya se está debatiendo internacionalmente acerca de la necesidad de crear mecanismos institucionales para regular las tecnologías de la IA (Robles Carrillo, 2020). Para Eduardo Magrani es importante reinterpretar el rol del derecho al diseñar soluciones normativas para la IA (Magrani, 2018). A grandes rasgos, existen dos grupos de posibles remedios para el uso negativo de la IA. El primero es que las futuras tecnologías sigan los estándares éticos, ya sea en la producción o en la aplicación. El primer grupo intenta crear una protección previa mediante dispositivos de seguridad. El segundo grupo de remedios aplica las reglas para proteger a los individuos contra los resultados posibles injustos de las aplicaciones de IA. Estas normas legales se enfocan en producir medios para que los individuos se opongan a las decisiones y para hacer cumplir las obligaciones de informar a los ciudadanos. Además, la premisa de los recursos legales es que no es posible determinar desde el inicio si la decisión de una aplicación de la computadora sería mejor o peor que una 
Alexandre Veronese et al Trayectoria normativa de la inteligencia artificial en los países de Latinoamérica con un marco jurídico para la protección de datos: límites y posibilidades de las políticas integradoras

decisión humana. Estas soluciones legales están presentes en muchos sistemas legales en un área que se amplía en el mundo: la ley de protección de los datos. En los artículos 13, 21 y 22 del Reglamento General de Protección de Datos (RGPD) de la Unión Europea se encuentran algunas disposiciones conocidas (Veronese et al., 2019) Muchos de los países Latinoamericanos construyeron algunas prescripciones legales de alguna manera.

Este artículo tiene dos secciones. Esta primera sección describe como Latinoamérica y los países del Caribe esperan dos olas de políticas públicas relacionadas con la protección de datos y la IA. La primera ola abarca los estatutos y sistemas administrativos para la protección de datos personales mientras que la segunda aprueba estrategias, planes y políticas nacionales para el desarrollo de la IA. Ambas tienen un origen indirecto en la difusión de las políticas públicas de los países, como el Mercado Único Digital de la Unión Europea. Aunque también está claro que otras políticas que no sean europeas pueden tener repercusión en la región. Las dos economías más grandes del mundo están en movimiento. Los Estados Unidos están coordinando el Grupo de Expertos en Recursos para la Investigación en Inteligencia Artificial (White House, 2021). Además, China planea lograr el liderazgo en la materia para el año 2030 (Roberts et al, 2020). Por consiguiente, la primera sección también describe la situación nacional de seis países en la región que poseen leyes de protección de datos y están desarrollando e implementando políticas públicas para la IA. Después de describir la convergencia potencial de estas dos políticas, el desarrollo de la IA y la protección de datos personales, la primera sección trabaja con un posible modelo de regulación para la IA. En esta primera sección también encontramos información de cada país acerca del desarrollo actual de estas políticas para obtener la aplicación posible de la ley de protección de datos para regular a la IA. Estos países son, especialmente, Colombia, México, Argentina, Uruguay, Chile, y Brasil.

La segunda sección también abre un debate sobre las posibilidades y los límites que conjugan la protección de datos con la IA. Se podría considerar que usar la prescripción de la protección de datos para regular a la IA sería muy difícil ya que algunas aplicaciones necesitarían acceso para que una gran cantidad de datos producen resultados predecibles. Otros pueden considerar que es posible utilizar prescripciones jurídicas para regular a la IA mediante la creación de nuevos derechos. Este estudio concluye que cualquier combinación de regulación efectiva demandará inversiones en innovación tecnológica para producir herramientas y por lo tanto resultados. También indica la necesidad de que los valores sociales estén insertos en las herramientas tecnológicas regulatorias.

La comparación de los modelos normativos en este artículo se basa en la metodología de revisión de la literatura creada por Marcio Iorio Aranha, la cual enfatiza la necesidad de integrar el análisis legal a los estudios institucionales. Establece que los principios legales son engranajes esenciales para entender el comportamiento de la revolución de la información en diferentes contextos nacionales, lo que aplica también a la inteligencia artificial y a su expansión (Iorio Aranha, 2011). Es decisivo mencionar, junto con este autor, que la literatura comparada usualmente no interrelaciona la descripción de las políticas con 
la evaluación de los marcos jurídicos. La originalidad de este estudio yace exactamente en la descripción comparada. Además, se puede evaluar que la metodología de Iorio Aranha es afín con la crítica clásica de la teoría social de Roberto Mangabeira Unger (Mangabeira Unger, 1976). Usualmente, las evaluaciones políticas o incluso la investigación académica son indiferentes al rol de la ley al diagnosticar los marcos sociales y políticos. En este estudio, no sucederá eso. Después de describir cada política nacional relacionada con la IA, habrá algunas consideraciones sobre la ley de protección de datos. El verdadero propósito de este método estará claro en la sección de debate. A la evolución de las políticas para fomentar a la IA la debe seguir la evolución del marco regulatorio, en el que la protección de datos puede ser un aspecto importante

\section{Estrategias, planes y políticas para la IA en Latinoamérica.}

La literatura acuerda que la IA tiene potencial para realizar grandes cambios en la sociedad y la economía del mundo. Las voces de Brasil lo señalan a través de una investigación publicada por colegas del Instituto de Estudios Avanzados para la Inteligencia Artificial (IA2) un consorcio de entidades brasileñas. Afirman que el ascenso de la IA puede tener un impacto social y económico más significante que la revolución de Internet y que las consideraciones éticas serán muy importantes (Cóbe et al., 2020). También desde Brasil, Fabrício Polido menciona el crecimiento exponencial de la IA en el campo de las políticas normativas y del derecho internacional y destaca las interacciones entre el derecho y la tecnología en el debate. Un informe patrocinado por la Universidad Stanford presenta una lista de varias estrategias nacionales en diferentes países (Bradley, Wingfield, and Metzger, 2020). Estas estrategias serían parte de lo que Tim Dutton llama la guerra para convertirse en líder en IA (Dutton, 2018). También, según la hipótesis de Andrés Ortega, este movimiento de producción estratégica sucede por la geopolítica de la cuarta revolución industrial. Brevemente debate el rol global de la UE en la búsqueda del dominio de la economía digital, con un incremento en el uso de la IA qué busca de la supremacía en esta discusión (Ortega, 2019). Federico Fernández-Montesinos, del Instituto Español de Estudios Estratégicos, comparte los mismos puntos de vista (Fernández-Montesinos, 2019). En breve, muchos investigadores y analistas se centrarán en entender a la IA como el nuevo centro de las agendas nacionales y regionales de las geopolíticas globales. Pero ¿qué significa esta regulación?

Como se mencionó con anterioridad, aquellas políticas públicas para la IA pueden estar relacionadas con la Ley de Protección de datos cuando se trata de encontrar soluciones a los usos injustos de estas aplicaciones. Por ejemplo, en la legislación de la UE, las antiguas directivas 95/46/CE tenían los mecanismos de control aplicables a la IA en los procesos de toma de decisión. Recientemente, la UE actualizó su sistema de protección de datos, cuando entró en vigor el RGPD (Reglamento

General de Protección de Datos) (Regulación UE 
2016/679). Esta regulación cuenta con la disposición legal del derecho a la información acerca del uso de los datos personales, lo cual se combina con el derecho de objetar los resultados del tratamiento de los datos personales (Veronese et al., 2019). El RGPD amplia en gran medida el derecho a oponerse al procesamiento de datos en comparación con la Directiva. Es importante destacar que la Regulación proviene de un Mercado Único Digital, entonces el derecho a la oposición es solo una porción de una política en construcción en la UE (Alexandre Veronese, 2019). Este movimiento normativo de la UE influenció, en cierta medida, en la redacción de la legislación nacional en lo concerniente a la protección de datos personales en los países Latinoamericanos. Por ejemplo, Chile ha tenido un estatuto sobre este tema desde 1999 (Chile, 1999). A pesar de ser el primero, Chile no tiene una entidad pública para hacer cumplir este marco. Esta es la razón principal por la cual Chile no puede tomar una decisión adecuada acerca de la Comisión Europea (Álvarez Valenzuela, 2016). Argentina fue el segundo país en obtener un estatuto en el tema en América Latina, el que se hizo ley en el año 2000 (Argentina, 2000). El debate argentino se basaba en ver si se podía usar el recurso de habeas data como una herramienta legal para acceder a los datos personales desde las bases de datos automatizadas. Algunos países de la región siguieron esta tendencia con la sanción de los estatutos nacionales desde principio hasta mediados de la década de 2000, como por ejemplo Uruguay en 2008 (Uruguay, 2008). El foco de estos estatutos cambió con el tiempo, a pesar de que todavía se debe hacer una mayor adaptación a las nuevas tecnologías. Estos dos últimos países, Argentina y Uruguay, son referencias para la región, porque la UE, en términos de datos personales, considera que tienen un nivel de protección adecuado. Otros estatutos nacionales más recientes, sin embargo, encajan en un contexto de un mayor potencial de nuevas tecnologías para crear mecanismos reguladores de la IA: México ha tenido su legislación de protección de datos personales desde 2010 (México, 2010) En Colombia, Perú, and República Dominicana los estatutos fueron promulgados ley en 2011, 2012, and 2013. También Costa Rica hizo lo mismo en 2011, así como también lo hicieron Brasil y Panamá en 2018 y 2019. La tabla a continuación resume el estado de la región

Tabla 1: Estado de las leyes de la protección de datos y autoridades administrativas para hacer valer los derechos en Latinoamérica. 


\begin{tabular}{|c|c|}
\hline Estado & Países, leyes y entidades administrativas \\
\hline $\begin{array}{l}\text { Países que tienen leyes } \\
\text { nacionales y autoridades } \\
\text { administrativas para hacer } \\
\text { valer los derechos de } \\
\text { protección de datos }\end{array}$ & $\begin{array}{l}\text { - Argentina (Ley Nacional No. } \\
\text { 25.326/2000, y Decreto Ejecutivo } \\
\text { Presidencial No. 1558/2001; “Dirección } \\
\text { Nacional de Protección de Datos } \\
\text { Personales / Agencia de Acceso a la } \\
\text { Información Pública”). } \\
\text { - Uruguay (Ley No. 18.331/2008, y } \\
\text { Decreto Ejecutivo no. 414/2009; “Unidad } \\
\text { Reguladora y de Control de Datos } \\
\text { Personales”). } \\
\text { - México ("Ley federal de protección de } \\
\text { datos personales en posesión de los } \\
\text { particulares", } 5 \text { de julio de 2010; } \\
\text { "Instituto Nacional de Transparencia, } \\
\text { Acceso a la Información y Protección de } \\
\text { Datos”). } \\
\text {-Perú (Ley No. 29.733/2011: “Dirección } \\
\text { General de Protección de Datos } \\
\text { Personales”). } \\
\text { - Costa Rica (Ley No. 8.969/2011: } \\
\text { “Agencia para la Protección de Datos } \\
\text { Personales”). } \\
\text { - Colombia (Ley No. 1.581/2012, y } \\
\text { Decreto Ejecutivo No. 1.377/2013: } \\
\text { "Superintendencia de Industria y } \\
\text { Comercio”); - Brasil (Ley Federal No. } \\
\text { 13.709/2018: “Autoridade Nacional de } \\
\text { Proteção de Dados”). } \\
\text { - Panamá (Ley No 81/2019: “Autoridad } \\
\text { Nacional de Transparencia y Acceso a la } \\
\text { Información”). }\end{array}$ \\
\hline $\begin{array}{l}\text { Países que tienen leyes } \\
\text { nacionales, pero que no } \\
\text { tienen la autoridad } \\
\text { administrativa } \\
\text { correspondiente para hacer } \\
\text { valer los derechos }\end{array}$ & $\begin{array}{l}\text { - Chile (Ley No. 19.628/1999). } \\
\text { - República Dominicana (Ley No. } \\
\text { 172/2013). }\end{array}$ \\
\hline
\end{tabular}

Sin embargo, más allá del panorama global y regional que muestra avances, las leyes nacionales latinoamericanas no consideran específicamente a la IA. En Latinoamérica, se 
están desarrollando planes, directivas y estrategias para implementar la IA en las políticas de desarrollo. Algunos países están más adelantados que otros. Más allá de lo mencionado, esta región todavía está en desventaja al compararse con los estados miembros de la UE, como se puede ver en el Informe anual de la Universidad de Stanford (Shoham et al, 2018). Este informe evalúa los indicadores de IA en varios campos alrededor del mundo y se ha estado haciendo por muchos años. (Zhang, 2021). Es el resultado de un gran estudio McKinsey con dos mil ciento treinta y cinco participantes, a quienes se les preguntó sobre la incorporación de las aplicaciones de IA en los países. El informe explica la ventaja de la UE en la IA por su compromiso con el tema. En la UE, este tema es parte de un conjunto amplio de políticas públicas, conocido como Mercado Digital Único. Existen otras evaluaciones, como el Índice de Preparación para la Inteligencia Artificial, Oxford Insights que mide la idoneidad de muchos países para implementar las aplicaciones IA (Oxford Insights, 2020). Este artículo mencionará esos índices nuevamente.

Se están realizando grandes esfuerzos para desarrollar la cooperación en este tema en la región. CEPAL trata de promover el rol principal en el tema de la IA en Latinoamérica y el Caribe para luchar por los procesos de integración de la política nacional en este contexto global. En este sentido, Márcio B. Braga presenta en su estudio publicado en 2002, dos fases del comportamiento de CEPAL en vista al desarrollo económico de Latinoamérica a través de la historia. En primer lugar, los países que hacían frente a problemas estructurales. La integración económica era la estrategia principal en la década de 1950. En la segunda fase, se focalizaron en la cooperación macroeconómica y la inserción de las economías de Latinoamérica en la economía internacional, ese período cubre los años 1990. (Bobik Braga, 2002). La primera fase abarca la integración económica regional como la «creación de un mercado común como respuesta necesaria a las demandas del proceso de crecimiento económico en la región, un proceso que se caracteriza por la industrialización que sustituye la importación.» (Braga, 2002) Esta fase no tuvo éxito por diversas razones. Algunos países desarrollaron las plataformas de industria nacional, mientras otros países no lo hicieron. Las sinergias potenciales en las economías regionales no fueron explotadas completamente. (Baer, 1972) La segunda fase de intento de integración se inspira en el proceso de integración de la UE con un concepto de «regionalismo abierto». Al leer los dos documentos recientes de CEPAL se entiende que una tercera fase está presente. El primero es el informe «Políticas de la industria y tecnología en Latinoamérica», de 2018 (CEPAL, 2017). El segundo informe es «El capital humano para la transformación digital en Latinoamérica», de 2019 (Katz, 2018). Es posible interpretar que esta tercera fase, de acuerdo con los objetivos de CEPAL, se concentra en la integración tecnológica. Dentro de este contexto, la IA surge como uno de los mecanismos disponibles para las políticas de desarrollo económico y social. Por lo tanto, sería razonable indicar que, en la actualidad, CEPAL busca fomentar la construcción de un «Mercado digital regional» para Latinoamérica. Muchos ejemplos de las iniciativas antes mencionadas - RedGEALC, IA Latam, OAS, y apoyo del BDIA, FairLAC - dan fundamento a estas imágenes.

Lo que preocupa en estas estrategias de IA nacional es la integración con la protección de 
los datos personales (Rotondo, 2020). La inserción de los principios de la transparencia de datos y la calidad en la construcción de las aplicaciones es parte de un sistema de protección posible. Después de todo, la solidez técnica es uno de los pasos necesarios para hacer valer la protección de los datos personales. La construcción de dispositivos de protección debe trascender a los discursos filosóficos y argumentativos sobre ética, seguridad, responsabilidad, justicia,» que estaban presente en aquellas estrategias, según Fabrício Polido (Polido, 2020). Es clave que las inversiones tecnológicas estén integradas a los temas de valores sociales.

Colombia, Argentina y Uruguay son algunos de los países que aparecen en el mapeo de la Organización para la Cooperación y el Desarrollo Económicos (OCDE) como aquellos que hacen esfuerzos reales para desarrollar políticas para la IA. Este análisis está a cargo del Observatorio de innovación en el sector público. Este proyecto cuenta con la participación de la OCDE y tiene el apoyo del Horizon 2020, un programa de la UE. La OCDE también intenta crear el Observatorio de Políticas de IA dentro de un contexto internacional en el que cerca de 50 países (incluyendo aquellos estados-miembro de la UE) han desarrollado o estén en el proceso de desarrollar las estrategias de IA nacional (OCDE, 2020). De las 50 iniciativas que la OCDE analiza, 37 focalizan en la IA para el sector público y tienen una mirada más amplia, también cubriendo la transformación digital en los gobiernos nacionales (OCDE, 2019). Los datos OCDE pueden combinarse con un informe de BDIA para dar una opinión más exacta de las políticas en Latinoamérica. Es importante mencionar que solo seis países están en el radar de BDIA realizando debates en el presente o en el futuro acerca de las estrategias (Gómez Mont et al, 2020).

En el mapeo OCDE de Latinoamérica se pueden ver cinco países: México, Colombia, Chile, Argentina, y Uruguay. Este mapa no muestra a Brasil, en claro contraste con el informe BDIA que también cubre este país:

Tabla 2. Estado de las seis estrategias de IA de Latinoamérica (completo o próximo). 


\begin{tabular}{|c|c|c|c|}
\hline & $\begin{array}{l}\text { Estado de la } \\
\text { estrategia } \\
\text { (OCDE)( } \\
\text { OCDE, 2020) }\end{array}$ & $\begin{array}{l}\text { Estado de la } \\
\text { estrategia BDIA } \\
\text { (Gómez Mont et } \\
\text { al, 2020) }\end{array}$ & $\begin{array}{l}\text { Relación con el sector } \\
\text { público (OCDE) } \\
\text { (OCDE, 2020). }\end{array}$ \\
\hline México & $\begin{array}{l}\text { Estrategia de } \\
\text { IA completa }\end{array}$ & $\begin{array}{l}\text { Continuidad a } \\
\text { explorar }\end{array}$ & $\begin{array}{l}\text { Incorporada a una } \\
\text { estrategia más amplia }\end{array}$ \\
\hline Colombia & $\begin{array}{l}\text { Estrategia de } \\
\text { IA próxima }\end{array}$ & Sí & $\begin{array}{l}\text { Rol público } \\
\text { reconocido, pero para } \\
\text { apoyar a los objetivos } \\
\text { del sector privado }\end{array}$ \\
\hline Chile & $\begin{array}{l}\text { Estrategia de } \\
\text { IA próxima }\end{array}$ & En progreso & $\begin{array}{l}\text { Incapaz de determinar } \\
\text { desde los materiales } \\
\text { públicos }\end{array}$ \\
\hline Argentina & $\begin{array}{l}\text { Estrategia de } \\
\text { IA próxima }\end{array}$ & $\begin{array}{l}\text { Continuidad a } \\
\text { explorar }\end{array}$ & $\begin{array}{l}\text { Incorporada a una } \\
\text { estrategia más amplia }\end{array}$ \\
\hline Uruguay & $\begin{array}{l}\text { Estrategia de } \\
\text { IA completa }\end{array}$ & Sí & Estrategia dedicada \\
\hline Brasil & No mencionada & En progreso & No mencionada \\
\hline
\end{tabular}

El tema es una nueva tendencia en la región. Por lo tanto, aún las estrategias «completas» de IA pueden tener varios grados de implementación y maduración. Para resolver este tema, los informes de BDIA evalúan muchos estudios de caso de cada país para ofrecer una evaluación general. Este estudio no reproduce las conclusiones del informe de BDIA. Su principal objetivo es evaluar que las leyes de IA orientadas hacia el uso responsable puedan, hasta cierto grado, confiar en el cumplimiento de la ley de protección de datos. Es claro que la IA puede otorgar las herramientas para mejorar los servicios públicos de Latinoamérica (Abdala, Lacroix Eussler, and Soubie, 2019). Es posible justificar las normas de la IA no solo para el crecimiento del patrimonio. Aunque sea razonable indicar que pueden surgir algunas amenazas por el uso no regulado de la IA. El origen de una de ellas puede ser la falta de transparencia en las decisiones de IA. Además, existen algunos riesgos que surgen del potencial sesgo del algoritmo, y el mal manejo de los datos de las materias. La recopilación de la información para completar el conjunto de datos puede plantear algunas cuestiones acerca de las consecuencias éticas de las variables seleccionadas. La tabla a 
continuación resume las posibilidades abstractas de regulación, como una tipología creada por los autores para este estudio.

Tabla 3. Posibles normas de IA.

\begin{tabular}{|c|c|c|}
\hline \multirow{2}{*}{ Fuente: } & \multicolumn{2}{|c|}{ Alcance de la regulación con ejemplos: } \\
\hline & General & Sectorial \\
\hline $\begin{array}{l}\text { Ley de } \\
\text { protección } \\
\text { de datos }\end{array}$ & $\begin{array}{l}\text { Una ley nacional que tiene } \\
\text { prescripciones por el uso } \\
\text { incorrecto de los datos } \\
\text { personales en las decisiones y } \\
\text { conjuntos de datos de IA } \\
\text { Ejemplo: Artículos } 21 \text { y } 22 \text { de } \\
\text { RGPD }\end{array}$ & $\begin{array}{l}\text { Las leyes específicas que cubren } \\
\text { algunas áreas de uso como la } \\
\text { seguridad nacional o las } \\
\text { actividades de los } \\
\text { consumidores. Ejemplo: el uso } \\
\text { legal de la ley del consumidor } \\
\text { para proteger a los ciudadanos } \\
\text { contra la discriminación en esas } \\
\text { relaciones. }\end{array}$ \\
\hline $\begin{array}{l}\text { Tablas } \\
\text { ética y } \\
\text { principios }\end{array}$ & $\begin{array}{l}\text { Un conjunto de reglas } \\
\text { abstractas que tienen como } \\
\text { objetivo a los desarrolladores } \\
\text { para fomentar la construcción } \\
\text { de aplicaciones de IA de } \\
\text { acuerdo con las reglas } \\
\text { generales éticas. Ejemplo: las } \\
\text { proposiciones de una } \\
\text { regulación general en el } \\
\text { debate del Parlamento de la } \\
\text { UE (European Commission, } \\
2020) \text {. }\end{array}$ & $\begin{array}{l}\text { La aplicación de prescripciones } \\
\text { éticas más o menos generales } \\
\text { solo para un determinado uso, } \\
\text { como los contratos laborales. } \\
\text { Ejemplo: la necesidad de todas } \\
\text { las contrataciones de IA que } \\
\text { fueron sometidas a un control } \\
\text { previo de solicitar una } \\
\text { reevaluación profunda hecha } \\
\text { por una junta de humanos. }\end{array}$ \\
\hline $\begin{array}{l}\text { Incentivos } \\
\text { económicos }\end{array}$ & $\begin{array}{l}\text { La propuesta de financiación } \\
\text { pública o privada a cualquier } \\
\text { tipo de proyecto de aplicación } \\
\text { de IA que cumpla con los } \\
\text { requisitos, ya sean posibles, } \\
\text { económicos, autosustentables } \\
\text { o prescripciones impulsadas } \\
\text { por la ética. Ejemplo: solo } \\
\text { permitir gastos en los } \\
\text { proyectos que tengan la } \\
\text { aprobación de la Junta de } \\
\text { Cumplimiento. }\end{array}$ & $\begin{array}{l}\text { La financiación de los proyectos } \\
\text { para aplicaciones de IA } \\
\text { principalmente cubre algunos } \\
\text { objetivos específicos, como } \\
\text { mejorar la seguridad local o } \\
\text { promover la actividad } \\
\text { económica. Ejemplo: concentrar } \\
\text { los esfuerzos de los gastos de IA } \\
\text { para promover una industria } \\
\text { elegida. Tratar de bloquear el } \\
\text { acceso a los fondos públicos por } \\
\text { algunas aplicaciones de IA. }\end{array}$ \\
\hline
\end{tabular}


Alexandre Veronese et al Trayectoria normativa de la inteligencia artificial en los países de

Latinoamérica con un marco jurídico para la protección de datos: límites y posibilidades de las políticas integradoras

La tabla anterior muestra el esfuerzo posible de los autores para reflejar las posibilidades de regular las aplicaciones de IA. Primero, es posible pensar en las normas sectoriales (salud, educación o seguridad, por ejemplo) para plasmar la especificidad de cada campo. Por otra parte, se puede pensar en la producción de unas normas más generales. Segundo, existen varias maneras de regular. Cualquier modelo normativo tendrá que manejar, al menos, las tres variables que están presentes en la tabla. Además, cualquier modelo normativo tendrá que combinar las variables en una política dinámica para producir el efecto deseado. Asimismo, es importante mencionar que tanto OCDE como BDIA clasifican a México y Uruguay como países con estrategias «completas». Se puede ser escéptico por esto por dos razones. La primera es que el tema todavía está en desarrollo, no solo en Latinoamérica sino alrededor del mundo. Es muy difícil de evaluar si los países tienen una política completa. Periódicamente, estas políticas y programas pedirán ajustes. Aún una «política completa» hipotética requerirá algunas modificaciones futuras mientras que se desarrolla la tecnología. El segundo concepto tiene relación con el concepto de liberación. ¿Es posible considerar a una política generada sin evaluar la efectividad? ¿Sobre qué estándares se puede medir? ¿La calidad del funcionamiento de las aplicaciones de IA? Por otra parte, ¿puede ser evaluado por la cantidad de recursos invertidos, o solo por los fondos públicos aplicados? Como se puede ver, estos informes son unas buenas hojas de ruta para comenzar el viaje. Sin embargo, este tema demandará muchas diferentes evaluaciones pronto.

Los siguientes subtemas describirán las seis iniciativas nacionales de los países mencionados con anterioridad, que forman parte de las políticas públicas en Latinoamérica. Junto con la descripción nacional, los subtemas mostrarán la información de la protección de datos aplicable a la IA. Además, los países que analizamos representan las primeras seis posiciones en el ranking Oxford Insights (Oxford Insights, 2020).

\subsection{Argentina}

En agosto de 2018, la Presidencia de la Nación implementó el plan nacional IA, que todavía estaba en desarrollo en esa fecha. (Argentina, 2018). En medio del proceso de creación inicial, se llevó a cabo la Conferencia sobre Inteligencia Artificial de la Secretaría de Modernización, del Ministerio de Producción y del Ministerio de Educación, Cultura, Ciencia y Tecnología (Argentina, 2019) El plan argentino focaliza en la competitividad del sector privado en IA para mejorar los servicios públicos. De acuerdo con el análisis OCDE, Argentina presentó una cuestión central en la innovación de IA y es un ejemplo en Latinoamérica. El plan es parte del amplio marco institucional en la Agenda Digital Argentina (Argentina, 2012) y los planes de Argentina Innovadora (Argentina, 2020). Todos se focalizan en los esfuerzos de planificación hasta alcanzar el 2030. El plan nacional original tiene tres ejes principales para aplicar el plan de IA: conocimiento, gobierno y sector productivo: En 2020, el gobierno otorgó a este plan el estado de «documento de referencia»

En Argentina, la sociedad civil ya reacciona ante lo relacionado con las aplicaciones de IA. 
La Asociación por los Derechos Civiles (ADC), una organización creada en 1995, que actúa en temas relacionados con el desarrollo tecnológico y la protección de los derechos, en especial en una perspectiva regional, ha publicado un material sobre la aplicación de los derechos humanos en el Plan Nacional de IA de Argentina (ADC, 2019). Este documento tiene una lista de catorce medidas que pueden aplicarse al plan de IA en lo relacionado con la privacidad, seguridad y protección de datos personales. Uno de ellos es la utilización de la ética y la privacidad durante el proceso para diseñar las aplicaciones (ética por diseño, o privacidad por diseño). Estos conceptos significan que, desde el diseño inicial de las nuevas tecnologías, deben cumplir con los estándares de protección internacional. Otro punto del texto de ADC es el pedido para clarificar el proceso de datos personales, especialmente en las técnicas de aprendizaje automático, la cual demanda un conjunto amplio de integración de datos. Otro punto de preocupación para ADC es el consentimiento informado, que requiere un mejor tratamiento realizado por el plan nacional de IA. El plan de IA apenas menciona la protección de datos. Sin embargo, son menciones muy generales (Argentina, 2020). Realmente, no existe una prescripción jurídica que reconozca el derecho de los ciudadanos a oponerse a una decisión automatizada en el estatuto nacional. Por otra parte, existe una propuesta para reformar el estatuto actual que puede convertir a este derecho en ley (Argentina, 2020).

No obstante, les preocupa específicamente el compromiso de las provincias del país de aplicar estos desarrollos con mayor transparencia en el proceso gradual de la implementación del plan. El diálogo entre los sectores públicos y privados, la sociedad civil, el mundo académico es otro aspecto abordado. De esta manera, aparece una perspectiva de multisectorial a su plan de IA Las reacciones no son una mera crítica. Abren un diálogo fructífero que ha desencadenado acciones decisivas, En junio de 2020, el Instituto de Tecnología de Massachusetts (MIT) dirigió la Cumbre de IA en Latinoamérica, la cual tuvo lugar en Argentina, para debatir sobre las oportunidades y riesgos del uso de IA en la región (OAC, 2020). Este breve debate solo muestra la necesidad de interrelacionar el plan de IA con futuras prescripciones jurídicas de protección de datos para crear un marco regulatorio sólido. De acuerdo con Oxford Insights, Argentina ocupa la cuarta posición en la región (Oxford Insights, 2020).

\subsection{Uruguay}

Este país se ha consolidado como uno de los líderes regionales en la incorporación de tecnologías de la información, incluidas dentro del suministro de servicio público. La World Wide Web Foundation presenta una investigación con muchos usos específicos de los algoritmos y la IA en el país y evidencia este crecimiento (World Wide Web Foundation, 2018). La Agencia de Gobierno Electrónico y Sociedad de la Información y del Conocimiento (AGESIC) es el cuerpo responsable para la Estrategia de Inteligencia Artificial para el Gobierno Digital. (Uruguay, 2019). En abril de 2019, publicaron un anteproyecto de normativa. La estrategia tiene como objetivo mejorar el funcionamiento del sector público y depende de cuatro pilares: la gestión de la IA en la Administración Pública, el desarrollo de 
Alexandre Veronese et al Trayectoria normativa de la inteligencia artificial en los países de

Latinoamérica con un marco jurídico para la protección de datos: límites y posibilidades de las

políticas integradoras

las capacidades, el uso responsable de la IA y la IA en la ciudadanía digital. La estrategia de IA es parte de la Agenda Digital Uruguay 2020 que recibió contribuciones de dos consultas públicas (Uruguay, 2019). El primer tema de la consulta fue acerca de los principios que abordan las estrategias de IA (Uruguay 2020). Los principios utilizados en la redacción de la estrategia eran el propósito, el interés general y la garantía de la inclusión y la equidad, respeto por los seres humanos, la transparencia, la responsabilidad, la ética, los beneficios, la privacidad por diseño y la seguridad. La consulta pública fue concluida en 2020. El informe del BDIA concluye que existe un «momento emprendedor» en fase de desarrollo y que el gobierno puede resaltarlo para acelerar, junto con la sociedad civil, una digitalización nacional, promoviendo la IA para el bienestar social (Gómez Mont et al, 2020).

De acuerdo con Oxford Insights, Uruguay es el país número cuarenta y cuatro en el mundo que desarrolla IA. Pero esta posición lo representa como el primer país en la región (Oxford Insights, 2020). La clasificación también muestra un resultado de cuatro principios relevantes para el uso responsable de la IA: privacidad, integración, transparencia y responsabilidad. En 2020, Uruguay actualizó la agenda digital aprobando «la Agenda Digital 2025- Sociedad Digital Resiliente» (Uruguay 2020). Esta nueva agenda tiene dos objetivos. Estos dos objetivos involucran a diferentes entidades administrativas. El marco regulatorio está regido por la «Agencia para el Desarrollo del Gobierno de Gestión Electrónica y la Sociedad de la Información y del Conocimiento» (AGESIC). La responsabilidad de la protección de datos recae en la «Unidad Reguladora y de Control de Datos Personales (URCDP).» Finalmente, Uruguay tiene una ley de protección de datos que directamente prescribe que los ciudadanos puedan oponerse a las decisiones automatizadas. Se halla en el artículo 16 de la ley de protección de datos (Uruguay, 2008). El mismo artículo prescribe el derecho de recibir información sobre el proceso de decisión de la IA. Esta prescripción jurídica es una medida válida que los ciudadanos pueden usar para protegerse contra las decisiones basadas en la IA que sean injustas o poco razonables.

\subsection{Colombia}

En noviembre de 2019, Colombia publicó su Política Nacional para la Transformación Digital y la Inteligencia Artificial de Colombia, un documento del Consejo Nacional de Política Económica y Social (CONPES) (Colombia, 2019). La política tiene como objetivo cumplimentar los requisitos de la transformación tecnológica de la OCDE. Colombia no solo es parte de esta organización internacional, sino que busca cumplir con las «Recomendaciones del Consejo en Inteligencia Artificial.» (OCDE, 2019). Jhon Caballero Martínez, de la Universidad Externado de Colombia, analizó el documento y lo comparó con la política de la OCDE. Se pregunta si esto podría ser el comienzo de una política pública efectiva en IA en el país (Martínez, 2019). La respuesta parece ser positiva ya que la política se integra al Plan de Desarrollo Nacional 2018-2022, (Colombia, 2018) y el uso de las nuevas tecnologías es uno de los componentes estratégicos del Sistema Nacional para la Competitividad y la Innovación de Colombia (Colombia, 2020). La SNCI es un gran proyecto de transformación digital de la economía y el sector público. La transformación digital en el 
Alexandre Veronese et al Trayectoria normativa de la inteligencia artificial en los países de

Latinoamérica con un marco jurídico para la protección de datos: límites y posibilidades de las políticas integradoras

sector público implica implementar un gobierno digital desde una política integrativa para desarrollar tareas institucionales utilizando nuevas tecnologías, como la IA. Este proceso se ha llevado a cabo en Colombia desde el 2008 en un programa que se llama «gobierno online» (Toro-García, Gutiérrez-Vargas, and Correa-Ortiz, 2020). El desafío más grande del proceso de transformación digital aplicado al gobierno es mostrar eficiencia y transparencia en la toma de decisiones de la autoridad pública.

En Colombia, existe una iniciativa privada en cuanto a la IA. Las estrategias del gobierno exigen que el sector público sea un aliado de las iniciativas privadas como regulador y facilitador de los servicios. En este sentido, la Política Nacional para la Transformación Digital y la Inteligencia Artificial de Colombia tiene cuatro objetivos específicos. El primero es bajar las barreras que evitan la incorporación de las tecnologías digitales en los sectores públicos y privados, para facilitar la transformación digital del país. El segundo es crear las condiciones que faciliten la innovación digital en los sectores privados y públicos, para convertirse en un mecanismo para el desarrollo de la transformación digital. La tercera es fortalecer las habilidades de los trabajadores para enfrentar los desafíos de la Cuarta Revolución Industrial. La cuarta es desarrollar las condiciones para facilitar la preparación del país para los cambios económicos y sociales que trae consigo la IA, así como también para estimular otras tecnologías innovadoras. La estrategia tiene catorce líneas de acción. Algunas de ellas abordan la creación de un mercado de la IA, construyen una estructura ética para la IA y finalmente alcanzan la interacción continua con la comunidad internacional y los expertos. Otra línea que merece ser mencionada es el rol crucial de la investigación académica en el desarrollo de un mercado de IA en Colombia (Gómez Mont et al., 2020). La protección de los datos contra las decisiones injustas de la IA en Colombia no está regulada por la ley de protección de datos. También se habla de la necesidad de actualizar la legislación de 2012 para abordar las decisiones automatizadas. (Dejusticia, 2019). Después de todo, aun la constitución del país utiliza un sistema de IA. (Velasco Fuentes et al, 2021) (Colombia, 2020).

En 2020, Colombia creó la Guía Ética para la Aplicación de IA en los sectores públicos y privados. El «Marco Ético para la Inteligencia Artificial en Colombia» (guías éticas) es un documento con principios y herramientas para desarrollar la IA (Colombia, 2020). El país, de acuerdo con la clasificación Oxford Insights, ocupa la tercera posición en la región (Oxford Insights, 2020). La Guía Ética puede ser importante para fortalecer el uso responsable de la IA en el país. Otro documento relevante publicado en 2021 viene del Consejo Internacional para la IA en Colombia. Alinea algunas respuestas institucionales para implementar una política de IA (Colombia, 2021).

\subsection{México}

En 2018, el gobierno federal publicó un estudio que contó con la colaboración de la Embajada del Reino Unido y los consultores Oxford Insights (Reino Unido) y C Minds (Colombia): «Hacia una estrategia de IA en México: Aprovechar la revolución de la IA» 
Alexandre Veronese et al Trayectoria normativa de la inteligencia artificial en los países de

Latinoamérica con un marco jurídico para la protección de datos: límites y posibilidades de las políticas integradoras

(México, 2018) Este documento sirve como base para la construcción de IA para este país. Este documento centraliza su análisis y otorga recomendaciones en cinco áreas. La primera es el gobierno y los servicios públicos. La segunda son los datos y la infraestructura digital. La tercera es la investigación y el desarrollo. La cuarta es la capacidad, habilidades y educación. La quinta es la ética. Como otros países de la región, la estrategia IA-MX 2018 encaja en la Estrategia Digital Nacional, que busca construir un México digital (México, 2013). El conjunto de marcos regulatorios que compone esta estrategia es el Ley Federal de Telecomunicaciones y Radiodifusión (México, 2014), el Plan Nacional de Desarrollo 2013-2018 (México, 2013), el Decreto Ejecutivo Nacional de Ventanilla Única (México, 2015), y el Decreto Ejecutivo sobre Datos Abiertos (México, 2015). Se realizó una consulta nacional a fines de 2018 para reunir contribuciones acerca de los derechos y desafíos de la transformación digital y la IA (México, 2020). La estrategia digital de México también cubre los cinco puntos principales para ser implementados antes de 2030 (IA2030Mx). Todos ellos vienen de principios y guías de 2018. El primero es crear una coalición de IA para promover los enfoques y los diálogos multisectoriales. El segundo es hacer un mapeo de usos y necesidades del sector y la identificación de las mejores prácticas gubernamentales. El tercero es fomentar el liderazgo internacional de México, enfatizar la OCDE y D9, un grupo que comprende nueve países (New Zealand, 2020). El cuarto es publicar las recomendaciones del informe producido después de la consulta pública. El quinto es trabajar en un subcomité de IA para proseguir los esfuerzos con los expertos y los ciudadanos. La Coalición IA2030Mx coordinó la primera Investigación Nacional en IA y está trabajando para desarrollar una propuesta de Estrategia Nacional de IA a través de los seis grupos temáticos: ética, gestión, gobierno y servicios públicos, investigación y habilidades de desarrollo, capacidades y educación, datos, infraestructura y ciberseguridad, y los ciudadanos mexicanos en el extranjero (Gómez Mont et al, 2020). El gobierno también creó iniciativas de datos abiertos como la Agencia Digital de Innovación Pública de la Ciudad de México (Agencia Digital de Innovación Pública - ADIP) (2018-2024). Además, es posible ver a México tanto en los avances federales como en las iniciativas locales, como ejemplo podemos citar a la agenda de IA en Jalisco con el foco en la innovación del gobierno y la IA. Existen otros ejemplos en el informe IADB de la cooperación entre los emprendedores y la sociedad civil, como el primer Centro IA para el bienestar social en el país (fAIr Jalisco) (Gómez Mont et al., 2020). Además, existen varias iniciativas en IA para resolver los problemas urbanos de la Ciudad de México (Puga et.al., 2021). De acuerdo con estas acciones, la BDIA concluye que México es una referencia en la región en lo relacionado con el desarrollo y la implementación de la IA para el bien social, a pesar de que el tema de la inclusión digital todavía sea un desafío para todo el país (Gómez Mont et al., 2020). De acuerdo con Oxford Insights, México ocupa el quinto lugar en la IA de Latinoamérica (Oxford Insights, 2020).

La mayoría de las leyes de protección de datos prescriben cuatro derechos generales, conocidos como «ARCO» (acceso, rectificación, cancelación y oposición) La ley federal de protección de datos mexicana (México, 2010) no prescribe un derecho claro para que los 
ciudadanos se oponen el tratamiento de los datos. Sin embargo, una persona puede interpretar la posibilidad de usar el derecho general de oposición contra el tratamiento de los datos (artículos 27 y 28) para protegerse a sí misma. No obstante, esa garantía jurídica demandaría cierta interpretación e información técnica sólida para probar que es efectiva en teoría. Se podría hacer si se siguieran los principios mencionados en el artículo 6. Pero no es posible oponerse a un tratamiento solamente con el argumento de que está automatizado. Tampoco es posible oponerse argumentando su injusticia. Para hacerlo, sería necesario probar algunas características ilegales del tratamiento. Esta situación legal se repite en algunos países como Perú y Colombia. Sin embargo, cuando se trata de la protección de datos en contra del gobierno, México tiene una ley diferente aprobada en 2017, y expresamente prescribe el derecho de oponerse a las decisiones automatizadas en el artículo 47 (II) (México, 2017).

\subsection{Chile}

El Ministerio de Ciencia, Tecnología; Conocimiento e Innovación (MCTCI) promulgó un documento titulado «Inteligencia artificial para Chile: El desarrollo urgente de una estrategia,» con sugerencias para las políticas de Chile. En 2019, el gobierno implementó un plan para desarrollar la «Política de IA Nacional de Chile» hasta 2020. La Comisión Nacional para la Investigación Tecnológica y Científica es el cuerpo responsable para la estrategia chilena. En febrero de 2020, Chile lanzó un proceso de consulta pública para recoger las percepciones y las preguntas de las organizaciones sociales y civiles, el mundo académico, el sector productivo y los ciudadanos en general acerca del uso y el desarrollo de la IA en el país. La idea era incorporar contribuciones a la política nacional hasta agosto de 2020 (Chile, 2020). Las contribuciones se integraron en los principales temas: posibles factores en el desarrollo de la IA y la aplicación, los aspectos éticos y regulatorios y los impactos sociales (Gómez Mont et al., 2020).

Otra forma de participación social de esta política de Chile es hacer un ciclo de varias reuniones temáticas. También podemos mencionar a la utilización de IA para la administración chilena a través de la sociedad pública-privada que implementó «Sofía,» un chatbox cognitivo que asiste a los nuevos emprendedores para comenzar nuevos negocios (Abdala). El MCTCI también creó un comité de expertos para elaborar un documento titulado «Hacia una estrategia I+D+i+S en IA para Chile» (Gómez Mont et al, 2020). De acuerdo con Oxford Insights, Chile es el segundo país en el desarrollo de la IA en Latinoamérica (Oxford Insights, 2020). En el 2020, Chile dio a conocer su política en materia de ciencia, tecnología, conocimiento e innovación, la que delinea objetivos generales para el desarrollo de nuevas tecnologías y otorga las guías del gobierno en el tema para el país (Chile, 2020).

Aún antes de las iniciativas gubernamentales, existieron movilizaciones de las sociedades civiles en lo relacionado con la ley de protección de datos e IA. En 2018, «Los Derechos Digitales» publicaron un informe titulado «Algoritmo y Desigualdad» para abogar por la 
necesidad de protección contra las decisiones exclusivamente automatizadas (Zuazo, 2018). En Chile, la protección de datos contra las decisiones de IA injustas no está regulada aún. Sin embargo, existen dos proyectos de ley («Boletín 11144-07 y 11092-07») para actualizarla ley vigente de la protección de datos (Chile). Cuando estos proyectos pasen a ser leyes, se creará el derecho y establecerá la autoridad administrativa de la protección de datos (Araya Paz, 2021).

Vale la pena señalar que, de los cinco países analizados, solo Uruguay no firmó las «Recomendaciones del Consejo en Inteligencia Artificial,» además de ser el único cuya estrategia ya tiene una versión final, de acuerdo con el cuerpo mismo de la investigación en el Observatorio de Innovación en el Sector Público de OCDE. Curiosamente, Uruguay es el país que ha llegado más lejos en la región con su plan de IA y tiene una ley de protección de datos que puede ofrecer medidas jurídicas de defensa para los ciudadanos contra las decisiones injustas o irrazonables. La primera parte la firmó como un Estado Miembro de la OCDE: México, Chile, y Colombia; que lo firmó antes de unirse a la OCDE. Una segunda parte firmada como no miembros: Argentina y Brasil.

\subsection{La estrategia de IA en Brasil}

En la actualidad, Brasil posee una estrategia de IA nacional diseñada por el Ministerio de Ciencia, Tecnología e Innovación. La estrategia estuvo sometida a la consulta pública entre el 12 de diciembre de 2019 y el 2 de marzo de 2020 (Brasil, 2020). La «Estrategia Brasileña de Inteligencia Artificial» representa la política básica para la introducción de nuevas tecnologías en el país, y da lugar a la creación de normas legales sectoriales, como el Decreto Ejecutivo de la Internet de las Cosas- decreto No 9.854 del 25 de junio de 2019, que presenta cuatro temas de discusión: industria, salud, ciudades inteligentes y agricultura. Las políticas brasileñas confían en un sistema más amplio, como las estrategias de los países analizadas con anterioridad. Existe un Decreto Ejecutivo en vigencia para otorgar una base legal al Sistema Nacional para la Transformación Digital, que constituye la estructura normativa para la gestión del desarrollo para la estrategia de IA: Decreto No 9.319, del 21 de marzo de 2018. El documento presenta tres ejes transversales: legislación, regulación y uso ético, aspectos internacionales y gestión de IA. Estos ejes se usan para los debates de consulta pública y plasman las preocupaciones por las inversiones en IA La estrategia de IA plantea cuestiones para crear barreras para desarrollar tales tecnologías y preservar los derechos de los usuarios, notablemente en lo relacionado con la protección de datos personales. Ana Claudia Farranha enfatiza que el futuro de los servicios públicos basados en la IA debe preservar los derechos de los usuarios y tal objetivo demanda la aplicación de los principios legales y éticos (Farranha, 2020). Entre los principios, los más importantes abordan la transparencia de los algoritmos. De acuerdo con la clasificación de Oxford Insights, Brasil se ubica sexto entre los mejores países en IA en la región, pero resulta ser el más bajo en transparencia en la clasificación del uso responsable (Oxford Insights, 2020). 
Todavía es necesaria la integración y la alineación entre la estrategia futura y las políticas públicas que ya existen sobre nuevas tecnologías, datos y gestión digital, como la «Estrategia de Transformación Digital Brasileña (E-digital), El Programa de Innovación Educativa Conectada, el Plan Nacional de Internet de las Cosas y la Estrategia Nacional para la Gestión Digital (e-gov). También es necesario conectar la IA a la entrada en vigor de la Ley General de Protección de Datos Personales de Brasil (LGPD, acrónimo en portugués), 1 a Ley de Derechos de Internet de Brasil (Marco Civil de Internet) y la Política de los Datos Abiertos (Decreto No. 8.771, de 2016). La construcción de la Autoridad Nacional de Protección de Datos de Brasil está avanzando lentamente. La ley de protección de datos brasileña incluye el derecho de objetar exclusivamente a las decisiones automatizadas en su artículo 20 (Brasil, 2018). Se cuestiona si entrará en vigencia (Rodríguez et al., 2020).

Diego Cortiz da Silva muestra que antes de estas iniciativas del gobierno en materia de la reglamentación de Internet y las iniciativas de transformación digital, el gobierno había creado en 2012 «El Programa Startup Brasil» Este programa trata de fomentar las prácticas emprendedoras de innovación a través de compañías tecnológicas pequeñas (Silva, 2015). En Brasil, los creadores de la IA son no solo las autoridades y compañías públicas, sino principalmente los investigadores y las instituciones académicas. El sector académico forma la columna vertebral de la construcción de la ciencia, la tecnología y la innovación. Los fondos primarios para la IA vienen directamente del presupuesto de investigación (Tadeu Arantes, 2019). Una fuente importante es CGI.br, la entidad que maneja el nombre de los dominios de la Internet en Brasil. La entidad está desarrollando diversas iniciativas para promover la IA y se publicó una versión preliminar de sus planes (NIC.br, 2019). Esta iniciativa de investigación apoya a las instituciones académicas, las que tienen un rol significativo en el desarrollo de IA.

\section{Discusión: el debate sobre las medidas jurídicas contra las decisiones automatizadas injustas o poco razonables}

El pedido de medidas éticas para regular la IA se ha extendido. Surgió un debate similar sobre los sistemas criptográficos (Rogaway, 2015). En este punto del estudio, está bastante claro que el uso de las aplicaciones de IA puede traer amenazas potenciales en contra de los derechos de los ciudadanos. Parece que solo la ley de protección de datos, en la actualidad, tiene el potencial de ofrecer algunas soluciones. A pesar de esto, se puede imaginar cuán complejo sería cambiar algunas situaciones que puedan surgir después de que la decisión entre en vigencia. Un ejemplo abstracto sería el uso de algoritmos para contratar personas para puestos laborales. Otro podría ser el uso de un algoritmo para decidir acerca de las cuestiones migratorias. Lo que estos ejemplos muestran es que cualquier solución para regular las aplicaciones de IA debería basarse en tres posibilidades mencionadas con anterioridad: prescripciones éticas, recursos legales e incentivos económicos junto con 
Alexandre Veronese et al Trayectoria normativa de la inteligencia artificial en los países de

Latinoamérica con un marco jurídico para la protección de datos: límites y posibilidades de las políticas integradoras

mecanismos de protección técnicos. Esos mecanismos de protección técnicos son fundamentales para el proceso. Cualquier evaluación de un sistema de IA de toma de decisión necesitaría expertos. El debate ético es muy importante. Sin embargo, no se puede ignorar la necesidad de expertos para ayudar en aquellas tres medidas normativas potenciales.

Algunas de las prescripciones jurídicas disponibles, en muchos países, tratan al derecho de objetar las decisiones automatizadas como una formalidad. El ciudadano puede oponerse a un resultado. Sin embargo, el resultado puede tener una lógica más transparente acerca de la decisión o el derecho de someterse a otra evaluación, ya sea por la aplicación basada en IA o por un humano. La evaluación de la igualdad y justicia en las decisiones automatizadas es un tema muy complejo y encaja en este punto neurálgico. Tal Z. Zarsky escribió un artículo muy interesante donde postula que RGPD no es compatible con la «era de Big Data» (Zarsky, 2017). El mismo razonamiento se aplica a las decisiones basadas en IA. Presenta cuatro ejemplos para evaluar la incompatibilidad del RGPD (Reglamento General de Protección de Datos) La primera es la limitación del propósito (artículo 5). Se sabe que los procesos contemporáneos de data-crunching [consiste en la preparación y modelización de un sistema o aplicación que se utiliza] utilizan una gran cantidad de datos no solo para fines concretos. El procesamiento de los datos de las aplicaciones de IA puede establecerse para encontrar nuevos aspectos o conclusiones. Por lo tanto, describe que una limitación previa del propósito podría impedir nuevas posibilidades de tratamiento de los datos, y podría, de esta manera, representar amenazas a start-ups y modelos de negocios. Además, reconoce algunas prescripciones jurídicas que tratan de atemperar este problema y aportan equilibrio a los nuevos tratamientos en los artículos 6 y 89 de RGPD. Sin embargo, son abstractos y complejos de manejar. La segunda incompatibilidad surge de un principio general de RGPD la minimización de datos (Artículos 5 y 25). Las aplicaciones de IA más innovadoras pueden usarse para inferir sobre resultados o predicciones inesperadas. El suministro mínimo de datos para alcanzar los resultados encaminados empobrecería los potenciales de IA predictiva. El tercer problema es la prescripción jurídica para limitar el procesamiento de los datos a algunas categorías, como raza, etnia, opiniones políticas, etc. (artículo 9) La razón es la misma que las otras dos. En el procesamiento de datos simple, esos límites no plantearían ninguna dificultad. Sin embargo, al trabajar con aplicaciones de IA más vanguardistas, esas limitaciones no solo son difíciles, sino que casi representan una desventaja para todo el rendimiento del tratamiento de datos. Finalmente, la cuarta inconsistencia es muy útil para el debate de este estudio. El autor considera que el artículo 22 de RGPD posee un claro riesgo en Big Data y que se puede extender para abarcar las aplicaciones de IA. Como fue descrito en este estudio, las prescripciones jurídicas les conceden a los ciudadanos el derecho de oponerse a las decisiones automatizadas, aun permitiendo su revisión por un procesamiento humano.

La solución legal a este problema no es simple. Sandra Wachtner y Brent Mittelstadt señalan la necesidad de crear un nuevo derecho a las decisiones automatizadas (Wachter, Mittelstadt, 2019). Ni el RGPD ni las leyes norteamericanas poseen el derecho de equidad 
Alexandre Veronese et al Trayectoria normativa de la inteligencia artificial en los países de

Latinoamérica con un marco jurídico para la protección de datos: límites y posibilidades de las políticas integradoras

contra los resultados del procesamiento de IA. Para ellos, el balance surgiría de otorgar a los ciudadanos un nuevo derecho de «cómo ser vistos». Compararon esta necesidad contemporánea al «derecho a ser olvidado» que el Tribunal de Justicia de la UE había reconocido. Sin embargo, no están exactamente seguros cómo un derecho se puede convertir en legislación de la UE. Se puede hacer una evaluación crítica de esta propuesta interesante para enfatizar la necesidad de más inversiones en soluciones técnicas para regular a la IA. Este razonamiento es contradictorio. Como mencionamos al principio de este estudio, los expertos y analistas están más preocupados en buscar soluciones éticas y legales para estos temas. Aunque, se puede adelantar que esas soluciones éticas o legales requerirán que se apliquen avances tecnológicos (Veronese et al., 2019).

Es por eso que los índices de evaluación de la inteligencia artificial son tan importantes. Se pueden mencionar nuevamente al Índice de Preparación para la Inteligencia Artificial de Oxford Insights y el índice HAI AI de Stanford University (Zhang, 2021). Midieron no solo el número de estudios publicados sobre la IA, sino el tipo de preparación de los países para permitir soluciones tecnológicas.

\section{Conclusión}

Es innegable la relevancia política que la IA tiene para fortalecer las economías nacionales. Sin embargo, es importante evaluar la posibilidad de una regulación efectiva de estas tecnologías en un escenario internacional. Esta razón intensifica la necesidad de desarrollar políticas regulatorias en el tema. Aunque de un modo local, las estrategias y planes poseen muchas similitudes y reflejan los puntos de vista internacionales como los de CEPAL, OCDE, RedGEALC, FairLAC, BDIA y OEA. Las estrategias de los países latinoamericanos tienen como objetivo crear un conjunto de principios y fundamentos para las aplicaciones de la IA con las soluciones relacionadas con la protección y los mecanismos de protección previos mediante la presentación de un conjunto de elementos éticos para la protección de datos con los que las tecnologías deben cumplir desde su diseño. Aun la inserción del derecho a objetar las decisiones automatizadas exclusivas está siendo defendido por la «Red Iberoamericana de Protección de Datos Personales». Esta red de protección de datos produjo un documento con los estándares actuales para la legislación contemporánea sobre la materia. [1]El artículo 29 prescribe el derecho de no ser afectado por las decisiones automatizadas exclusivamente (REDIPD, 2017). Por lo tanto, el panorama regional está claramente evolucionando. Sin embargo, estos mecanismos no son suficientes. Después de todo, el contenido de una decisión tomada por una computadora no está definido previamente. Algunas soluciones alternativas son complementarias y necesarias. Un modo es incorporar mecanismos para disminuir el potencial de impactos negativos mediante el fortalecimiento de la protección de datos personales Un modo de hacerlo es incorporar la protección de datos personales en las iniciativas de la regulación de la IA entre las acciones para las transformaciones digitales de los países. Por lo tanto, con la combinación de los mecanismos de protección previos y las prescripciones jurídicas para otorgar protección 
será posible crear un marco regulatorio para minimizar los riesgos. Las estrategias nacionales de IA van más allá de los planes de inversión. Solo lograrán su potencial una vez que se discuta sobre una sinergia entre el desarrollo de proyectos tecnológicos de los jugadores centrales y evalúen las consecuencias de estos mecanismos en los campos económicos, sociales y legales.

\section{Referencias bibliográficas}

- ADC, "Una Perspectiva de Derechos para el Plan Nacional de Inteligencia Artificial,” Asociación por los Derechos Civiles (ADC, July 18, 2019), https://adc.org.ar/2019/07/18/una-perspectiva-de-derechos-para-el-plan-nacional-de-intelige ncia-artificial/.

- Alexandre Veronese, "The right of explanation and the right to object automatic decisions: comparing the European Union General Data Protection Regulation (EU/GDPR) with the Brazilian General Data Protection Federal Act (BGDPFA)," CPRLATAM Conference, Cordoba, Argentina, July 1-2, 2019, in coordination with CLT2019, July 1-5, 2019, pp.125-134.

- Alexandre Veronese, Alessandra Silveira, and Amanda Nunes Lopes Espiñeira Lemos, "Artificial intelligence, Digital Single Market and the Proposal of a Right to Fair and Reasonable Inferences: A Legal Issue between Ethics and Techniques," UNIO: EU Law Journal, vol. 5, no. 2 (2 July 2019): pp. 75-91, https://doi.org/10.21814/unio.5.2.2294.

- Ana Claudia Farranha, "Serviços Públicos e Estratégia Brasileira de Inteligência Artificial: Primeiras Abordagens," in Derecho, Inteligencia Artificial y Nuevos Entornos Digitales (Seville: Asociación Andaluza de Derecho, Medio Ambiente y Desarrollo Sostenible, 2020), pp. 93-104.

- Andrés Ortega, “Geopolítica de la Cuarta Revolución Industrial,” Economistas, no. 165 (October, 2019): pp. 21-24, https://www.cemad.es/wp-content/uploads/2019/10/Geopolitica-4RI.pdf, 23.

- Andrés Toro-García, Cristian Gutiérrez-Vargas, and Luis Correa-Ortiz, “Estrategia de Gobierno Digital para la Construcción de Estados Más Transparentes y Proactivos," Trilogía Ciencia Tecnología Sociedad 12, no. 22 (January 31, 2020): pp. 71-102, https://doi.org/10.22430/21457778.1235.

- Carlos Araya Paz, "Transparencia Algorítmica ¿Un Problema Normativo o Tecnológico?” CUHSO (Temuco). Epub (2021), https://scielo.conicyt.cl/scielo.php?pid=S2452-610X2021005000002\&script=sci_arttext.

- CEPAL. "Políticas Industriales y Tecnológicas en América Latina,” CEPAL (CEPAL, Santiago, November 2017).

https://www.cepal.org/es/publicaciones/42363-politicas-industriales-tecnologicas-america-lat 
Alexandre Veronese et al Trayectoria normativa de la inteligencia artificial en los países de Latinoamérica con un marco jurídico para la protección de datos: límites y posibilidades de las

ina.

- Charles Bradley, Richard Wingfield, and Richard Metzger, "National Artificial Intelligence Strategies and Human Rights: A Review," Global Partners Digital (Stanford's Global Digital Policy Incubator, Stanford, CA, April 15, 2020),

https://www.gp-digital.org/publication/national-artificial-intelligence-strategies-and-human-r ights-a-review/.

- Constanza Gómez Mont et al., "Artificial Intelligence for Social Good in Latin America and the Caribbean: The Regional Landscape and 12 Country Snapshots. " (Washington, DC:

IADB, C Minds, July 2020),

https://publications.iadb.org/en/artificial-intelligence-for-social-good-in-latin-america-and-the -caribbean-the-regional-landscape-and-12-country-snapshots.

- Daniel Álvarez Valenzuela. “Acceso a la información pública y protección de datos personales ¿puede el Consejo para la Transparencia ser la autoridad de control en materia de protección de datos?" Revista de Derecho Universidad Católica del Norte, vol. 23, no. 1 (2016): pp. 51-79, https://scielo.conicyt.cl/scielo.php?script=sci_abstract\&pid=S0718-97532016000100003\&ln $\mathrm{g}=\mathrm{es} \& \mathrm{nrm}=\mathrm{iso}$.

- Dejusticia, “Colombia: en Mora de Actualizar la Protección de DatosPpersonales para la Era Digital", (2019),

https://www.dejusticia.org/colombia-en-mora-de-actualizar-la-proteccion-de-datos-personale s-para-la-era-digital.

- Diogo Silva, "Conhecimento, tecnologia e futuro: Análise do Cenário de Inovação dos Países Emergentes," (Teses e Dissertações dos Programas de Pós-Graduação da PUC-SP, São Paulo, 2015), https://tede2.pucsp.br/handle/handle/18180.

- Eduardo Magrani. "Governance of Internet of Things and Ethics of Artificial Intelligence." Revista Direitos Culturais. Santo Ângelo, vol. 13, no. 31 (Set - Dec.2018), pp.153-190, http://dx.doi.org/10.20912/rdc.v13i31.2816.

- European Commission, "Proposal for a Regulation of the European Parliament and of the Council laying down harmonized Rules on Artificial Intelligence (Artificial Intelligence Act) and Amending certain Union Legislative Acts, COM/2021/206 final," (Brussel, 2020), https://digital-strategy.ec.europa.eu/en/library/proposal-regulation-laying-down-harmonisedrules-artificial-intelligence.

- Fabrício Polido, "Inteligência Artificial entre Estratégias Nacionais e a Corrida Regulatória Global: Rotas Analíticas para uma Releitura Internacionalista e Comparada,” Revista da Faculdade de Direito da UFMG, no. 76 (2020): pp. 229-256, https://doi.org/10.12818/p.0304-2340.2020v76p229, 233.

- Felipe Calderon-Valencia, Juan-Jose Perez-Montoya, Fausto Santos de Morais, "Sistemas 
de IA en la Experiencia del Supremo Tribunal Federal Brasileño y la Corte Constitucional Colombiana," The Law, State and Telecommunications Review, vol. 13, no. 1 (May 2021): pp. 143-169, https://periodicos.unb.br/index.php/RDET/article/view/35614/29761.

- Felipe Rotondo, "Inteligencia Artificial, Protección de Datos Personales y Administración Pública," in Derecho, Inteligencia Artificial y Nuevos Entornos Digitales (2020), pp. 115-130.

- Federico Fernández-Montesinos, “La Inteligencia Artificial como Factor Geopolítico,” bie3. Boletín IEEE, no. 14 (2019): pp. 262-281, https://dialnet.unirioja.es/servlet/articulo?codigo $=7264320$.

- Huw Roberts et al., "The Chinese Approach to Artificial Intelligence: An Analysis of Policy, Ethics, and Regulation," AI \& Society, vol. 36, pp. 59-76, (17 June 2020), https://link.springer.com/article/10.1007/s00146-020-00992-2.

- IA Latam, “About us,” (2021), https://ia-latam.com.

- Jhon Martínez, “CONPES 3975 De 2019: ¿El Principio de una Política Pública sobre Inteligencia Artificial En Colombia?” Departamento de Derecho Informático (Universidad Externado de Colombia), (2019), https://derinformatico.uexternado.edu.co/conpes-3975-de-2019/.

- Jorge Puga et.al., “Inteligencia Artificial en México: Construyendo una Agenda,” (2020), https://educacion.nexos.com.mx/inteligencia-artificial-en-mexico-construyendo-una-agenda/, accessed June 16, 2021.

- José Tadeu Arantes, “FAPESP Criará Oito Centros de Pesquisa em Inteligência Artificial com o Governo Federal,” (Agência FAPESP, São Paulo) (2019), https://agencia.fapesp.br/fapesp-criara-oito-centros-de-pesquisa-em-inteligencia-artificial-co m-o-governo-federal/32196/.

- Katitza Rodriguez et.al., “Un Panorama Retrospectivo y Futuro de la Protección de Datos en América Latina y España,” Eletronic Frontier Foundation, (2020), https://www.eff.org/es/deeplinks/2020/09/look-back-and-ahead-data-protection-latin-america -and-spain.

- Loren Moss, "Organization of American States Redoubles Efforts to Combat Corruption with Big Data, Artificial Intelligence \& Analytics," Finance Colombia (13 August 2019), https://www.financecolombia.com/organization-of-american-states-redoubles-efforts-to-comb at-corruption-with-big-data-artificial-intelligence-analytics.

- Márcio Bobik Braga, "Integração Econômica Regional na América Latina: Uma Interpretação das Contribuições da Cepal," Brazilian Journal of Latin American Studies 1, no. 1 (December 1, 2002): pp. 1-26, https://doi.org/10.11606/issn.1676-6288.prolam.2002.81745. 
- Márcio Iorio Aranha, “Diálogo Político-Jurídico na Comparação de Modelos Regulatórios de Comunicação,” Revista Brasileira de Políticas de Comunicação, vol.1, (2011), pp. 1-20. https://repositorio.unb.br/bitstream/10482/9638/1/ARTIGO_DialogoPoliticoJuridico.pdf.

- Margarita Robles Carrillo, "La Gobernanza de la Inteligencia Artificial: Contexto y parámetros generales," Revista Electrónica de Estudios Internacionales, no. 39 (June 2020): pp. 1-27, https://doi.org/10.17103/reei.39.07.

- María Belén Abdala, Santiago Lacroix Eussler, and Santiago Soubie, "La Política de la Inteligencia Artificial: Sus Usos en el Sector Público y sus Implicancias Regulatorias," (CIPPEC, 2019),

https://www.cippec.org/wp-content/uploads/2019/10/185-DT-Abdala-Lacroix-y-Soubie-La-pol \%C3\%ADtica-de-la-Inteligencia-Artificial-octubre-2019.pdf.

- Natalia Zuazo, “Algoritmos y Desigualdades,” Derechos Digitales (2018), https://www.derechosdigitales.org/wp-content/uploads/algoritmo_desigualdad_cast.pdf

- NIC.br, "Plano de Trabalho do NIC.br na Área de Inteligência Artificial," CGI.br Comitê Gestor da Internet no Brasil (2019), https://www.cgi.br/media/atas/Iniciativa-NIC.br-IA.pdf.

- OAC, “Cumbre de Inteligencia Artificial Latinoamericana en MIT,” OAC: boletín de enero febrero 2020 (Observatorio Argentino del Ciperespecio, Buenos Aires, 2020), http://190.12.101.91/jspui/bitstream/1847939/1356/1/2020\%2001\%20BOLETIN\%2020\%200 AC.pdf.

- OECD, “AI Strategies; Public Sector Components," Observatory of Public Sector Innovation, Paris (2020) https://oecd-opsi.org/projects/ai/strategies.

- OECD, "Hello, World! Artificial Intelligence and Its Use in the Public Sector" (OECD Working Papers on Public Governance, Paris, November 2019), https://oecd-opsi.org/wp-content/uploads/2019/11/AI-Report-Online.pdf.

- OECD, "Recommendation of the Council on Artificial Intelligence," OECD Legal Instruments, Paris, May 21, (2019), https://legalinstruments.oecd.org/en/instruments/OECD-LEGAL-0449.

- Oxford Insights, “AI Readiness Index 2020, " (Oxford, Oxford Insights, 2020), https://www.oxfordinsights.com/government-ai-readiness-index-2020.

- Patrício Velasco Fuentes et. al., “Decisiones Automatizadas en la Función Pública en América Latina: Una Aproximación Comparada a su Aplicación en Brasil, Chile, Colombia y Uruguay," Derechos Digitales (2021), https://www.apc.org/sites/default/files/CPC_informeComparado.pdf.

Perú, “Ley no 29.733: Ley de Protección de Datos Personales,” (El Peruano, Lima, July 3, 2011). https://leyes.congreso.gob.pe/Documentos/Leyes/29733.pdf. 
- Phillip Rogaway, "The Moral Character of Cryptographic Work,” (University of California Davis, 2015), https://web.cs.ucdavis.edu/ rogaway/papers/moral-fn.pdf.

- Raphael Cóbe et al., "Rumo a uma Política de Estado para Inteligência Artificial,” Revista USP, no. 124 (March 19, 2020): pp. 37-48,

https://doi.org/10.11606/issn.2316-9036.v0i124p37-48.

- Raúl Katz, “Capital Humano para la Transformación Digital en América Latina,” Comisión Económica para América Latina y el Caribe (March 2018),

https://www.cepal.org/es/publicaciones/43529-capital-humano-la-transformacion-digital-ame rica-latina.

- REDIPD, “Red Iberoamericana de Protección de Datos Personales,” (2021), https://www.redipd.org/es.

- REDIPD. “Estándares de Protección de Datos Personales para los Estados Iberoamericanos," (2017), https://www.redipd.org/es/documentos/estandares-iberoamericanos.

- Roberto Mangabeira Unger, "Law in Modern Society: Towards a Criticism of Social Theory," New York, NY, Free Press (1976).

- Sandra Wachter, Brent Mittelstadt, "Right to Reasonable Inferences: Re-thinking Data Protection Law in the Age of Big Data and AI, " Columbia Business Law Review, vol. 2 (2019): pp. 494-620, http://robotic.legal/wp-content/uploads/2018/09/SSRN-id3248829.pdf

- Tal Z. Zarsky, "Incompatible: the GDPR in the Big Data Age, " Seton Law Review vol. 47, no. 4 (2017): pp. 995-1020, https://scholarship.shu.edu/shlr/vol47/iss4/2/.

- Tim Dutton, “An Overview of National AI Strategies,” Medium, n.p., (July 28, 2018), https://medium.com/politics-ai/an-overview-of-national-ai-strategies-2a70ec6edfd.

- Werner Baer, "Import Substitution and Industrialization in Latin America: Experiences and Interpretations.” Latin American Research Review vol. 7, no. 1 (1972): pp. 95-122, https://www.jstor.org/stable/2502457.

- White House, "The Biden Administration Launches the National Artificial Intelligence Research Resource Task Force," Washington, DC: White House's Press Room (10 June 2021),

https://www.whitehouse.gov/ostp/news-updates/2021/06/10/the-biden-administration-launch es-the-national-artificial-intelligence-research-resource-task-force.

- World Wide Web Foundation. “Algoritmos e Inteligencia Artificial en Latinoamérica," (2018), https://webfoundation.org/docs/2018/09/WF_AI-in-LA_Report_Spanish_Screen_AW.pdf.

- Yoav Shoham et al., "The AI Index 2018 Annual Report,” Stanford, CA: AI Index Steering 
Committee, (2018).

- Daniel Zhang, Saurabh Mishra, Erik Brynjolfsson, John Etchemendy, Deep Ganguli, Barbara Grosz, Terah Lyons, James Manyika, Juan Carlos Niebles, Michael Sellitto, Yoav Shoham, Jack Clark, and Raymond Perrault, "The AI Index 2021 Annual Report," AI Index Steering Committee, Human-Centered AI Institute, Stanford University, Stanford, CA, March 2021.

\section{Legislación y reportes nacionales citados}

\section{Argentina}

- Argentina, “Ley 25326/2000: Protección de los Datos Personales,” Argentina.gob.ar, Buenos Aires, November 2, 2000, https://www.argentina.gob.ar/normativa/nacional/ley-25326-64790/texto.

- Argentina, “Plan Nacional de Inteligencia Artificial,” Universidad Abierta Interamericana (Presidencia de la Nación, Buenos Aires, August 2018), https://www.uai.edu.ar/ciiti/2019/buenos-aires/downloads/B1/JA-Plan-Nacional-IA.pdf.

- Argentina, "Plan Nacional de Inteligencia Artificial, " (Buenos Aires, Presidencia de la Nación, 2020),

https://ia-latam.com/wp-content/uploads/2020/09/Plan-Nacional-de-Inteligencia-Artificial.pdf

- Argentina, “Proyecto de Ley - Ley de Protección de Datos Personales,” (Buenos Aires, Camara de Diputados, 2020), https://www4.hcdn.gob.ar/dependencias/dsecretaria/Periodo2020/PDF2020/TP2020/6234-D2020.pdf.

- Argentina, “Agenda Digital Argentina - Decreto 996/2018,” Boletín Oficial de la República Argentina, Buenos Aires, November 2, 2012, https://www.boletinoficial.gob.ar/detalleAviso/primera/195154/20181105.

- Argentina, “Argentina Innovadora 2030: Plan Argentina Innovadora 2020," Argentina.gob.ar, Buenos Aires, 2020, https://www.argentina.gob.ar/ciencia/argentina-innovadora-2030/plan-argentina-innovadora2020.

- Argentina, “Desconferencia sobre Inteligencia Artificial: En el Marco del Proceso de Creación del Plan Nacional de Inteligencia Artificial, se Realizó un Encuentro sobre la Temática en el Centro Cultural de la Ciencia," Argentina.gob.ar, Buenos Aires, July 5, 2019, https://www.argentina.gob.ar/ciencia/desconferencia-sobre-inteligencia-artificial.

\section{Brasil}


- Brasil, "Consulta Pública: Estratégia Brasileira de Inteligência Artificial," participa.br, n.p., 2020, http://participa.br/profile/estrategia-brasileira-de-inteligencia-artificial.

- Brasil, “Decreto 9.854, de 25 de junho de 2019," planalto.gov.br, n.p., June 25, 2019, http://www.planalto.gov.br/ccivil_03/_Ato2019-2022/2019/Decreto/D9854.htm.

- Brasil, “Lei no 13.709/2018: Lei Geral de Proteção de Dados Pessoais,” planalto.gov.br (Presidência da república, Brasília, August 15, 2018), http://www.planalto.gov.br/ccivil_03/_ato2015-2018/2018/lei/L13709.htm.

\section{Chile}

- Chile, "Ley 19628: sobre Protección de la Vida Privada,” Biblioteca del Congresso Nacional de Chile, n.p., August 18, 1999, https://nuevo.leychile.cl/navegar?idNorma=141599.

- Chile, "Política Nacional de Ciencia, Tecnología, Conocimiento e Innovación." (Ministerio de Ciencia, Tecnología, Conocimiento e Innovación). 2020, https://ia-latam.com/portfolio/politica-de-ciencia-tecnologia-conocimiento-e-innovacion-chile/

- Chile, "Proceso de Participación para Contribuir con la Política Nacional de Inteligencia Artificial,” n.p., 2020, http://www.minciencia.gob.cl/Pol\%C3\%ADticaIA.

- Chile, "Proyecto de Ley Boletín 11144-07 y 11092-07. Regula la protección y el tratamiento de los datos personales y crea la Agencia de Protección de Datos Personales," Senado, https://www.senado.cl/appsenado/templates/tramitacion/index.php?boletin_ini=11144-07.

\section{Colombia}

- Colombia, “Consejo Internacional de Inteligencia Artificial para Colombia,” (Gobierno de Colombia y Banco de Desarollo de America Latina, 2021), https://dapre.presidencia.gov.co/TD/CONSEJO-INTERNACIONAL-INTELIGENCIA-ARTIFICI AL-COLOMBIA.pdf

- Colombia, “Ley Estaturaria 1581 de 2012,” Función Pública (Gestor Normativo, Bogotá, October 17, 2012),

https://www.funcionpublica.gov.co/eva/gestornormativo/norma.php?i=49981.

- Colombia, "Marco Ético para la Inteligencia Artificial en Colombia," (Gobierno de Colombia, Consejería Presidencial para asuntos económicos y transformación digital y Banco de Desarrollo de América Latina). 2020, https://dapre.presidencia.gov.co/AtencionCiudadana/DocumentosConsulta/consulta-marco-et ico-IA-Colombia-200813.pdf

- Colombia, “Plan Nacional de Desarrollo 2018-2022: Pacto por Colombia, Pacto por la Equidade,” DNP, Bogotá, 2018, 
https://colaboracion.dnp.gov.co/CDT/Prensa/Resumen-PND2018-2022-final.pdf.

- Colombia, "Política Nacional para la Transformación Digital e Inteligencia Artificial,”

MinTIC (CONPES, n.p., November 8, 2019),

https://www.mintic.gov.co/portal/604/articles-107147_recurso_1.pdf.

- Colombia, "Sistema Nacional de Competitividad e Innovación - SNCI," Colombia

Competitiva (SNCI, Bogotá, 2020), http://www.colombiacompetitiva.gov.co/snci.

\section{Costa Rica}

- Costa Rica, “Ley no 8.968: Ley de Protección de la Persona Frente al Tratamiento de sus Datos Personales," Procuradoría General de la República (SCU, San José, July 7, 2011), http://www.pgrweb.go.cr/scij/Busqueda/Normativa/Normas/nrm_texto_completo.aspx?param 1=NRTC\&nValor1=1\&nValor2=70975\&nValor3=85989\&strTipM=TC.

\section{Mexico}

- Mexico, “Decreto por el que se Establece la Regulación en Materia de Datos Abiertos,” DOF (Diario Oficial de la Federación, Mexico City, February 20, 2015),

http://dof.gob.mx/nota_detalle.php?codigo $=5382838$.

- Mexico, "Decreto por el que se Establece la Ventanilla Única Nacional para los Trámites e Información Del Gobierno" (Diario Oficial de la Federación, Mexico City, February 3, 2015), https://www.dof.gob.mx/nota_detalle.php?codigo $=5380863$.

- Mexico, “Encuesta Nacional de IA,” Ia2030mx n.p., accessed July 5, 2020, https://www.ia2030.mx/consulta.

- Mexico, “Estrategia Digital Nacional: Mexico Digital,” (Gobierno de Mexico, n.p., November 2013), https://www.gob.mx/mexicodigital.

- Mexico, "Hacia una Estrategia de IA en México: Aprovechando la Revolución de la IA," (Mexico City, June 2018), https://docs.wixstatic.com/ugd/7be025_ba24a518a53a4275af4d7ff63b4cf594.pdf.

- Mexico, “Ley Federal de Protección de Datos Personales en Posesión de Los Particulares,” Camara de Diputados (Diario Oficial de la Federación, Mexico City, July 5, 2010), http://www.diputados.gob.mx/LeyesBiblio/pdf/LFPDPPP.pdf.

- Mexico, "Ley General de Protección de Datos Personales en Posesión de Sujetos Obligados," Camara de Diputados (Diario Oficial de la Federación, Mexico City, January 26, 2017), http://www.diputados.gob.mx/LeyesBiblio/pdf/LGPDPPSO.pdf

- Mexico, “Ley Federal de Telecomunicaciones y Radiodifusión,” Camara de Diputados (Diario Oficial de la Federación, July 14, 2014), http://www.diputados.gob.mx/LeyesBiblio/pdf/LFTR_240120.pdf. 
- Mexico, "Plan Nacional de Desarollo 2013-2018," (Diario Oficial de la Federación, Mexico City, May 20, 2013),

http://www.dof.gob.mx/nota_detalle.php?codigo=5299465\&fecha=20/05/2013.

\section{New Zealand}

- New Zealand, "The Digital 9," New Zealand Digital government, Wellington, (July 1, 2020), https://www.digital.govt.nz/digital-government/international-partnerships/the-digital-9.

\section{Panamá}

- Panama, "Ley no 81, de 26 de marzo de 2019, sobre protección de datos personales," ANTAI (Gaceta Oficial Digital, Ciudad de Panamá, March 29, 2019), https://www.antai.gob.pa/wp-content/uploads/2019/04/Ley-81-de-2019-Proteccion-de-DatosPersonales.pdf.

República Dominicana

- República Dominicana, “Ley no 172/2013, de 13 de diciembre de 2013,” (Gaceta Oficial, Santo Domingo, December 15, 2013), https://indotel.gob.do/media/6200/ley_172_13.pdf.

\section{Uruguay}

- Uruguay, "Agenda Uruguay Digital 2025 - Sociedad digital resiliente," (Presidencia de la República Oriental del Uruguay, Agencia de Gobierno Electrónico y Sociedad de la Información y del Conocimento, n.p.), accessed June 15, 2021, https://www.gub.uy/uruguay-digital/comunicacion/publicaciones/agenda-uruguay-digital-202 5-sociedad-digital-resiliente/agenda-uruguay-0

- Uruguay, "Consulta Pública Estrategia Nacional de Inteligencia Artificial para el Gobierno Digital," Camara Uruguaya de Tecnologías de la Información, n.p., 2019, https://www.cuti.org.uy/novedades/1199-consulta-publica-estrategia-nacional-de-inteligencia -artificial-para-el-gobierno-digital.

- Uruguay, "Estrategia de Inteligencia Artificial para el Gobierno Digital," gub.uy (Presidencia de la República Oriental del Uruguay, Montevideo, 2019), https://www.gub.uy/system/documents/attachments/000/000/003/original/0177491ab68dc7b 819694c237a705f4a7ba95e73.pdf.

- Uruguay, "Ley 18.331: Ley de Protección de Datos Personales," IMPO (La Dirección Nacional de Impresiones y Publicaciones Oficiales, Montevideo, August 18, 2008), https://www.impo.com.uy/bases/leyes/18331-2008.

- Uruguay, "Principios Generales sobre Inteligencia Artificial para el Gobierno Digital," gub.uy (Presidencia de la República Oriental del Uruguay, Agencia de Gobierno Electrónico y Sociedad de la Información y del Conocimento, n.p.), accessed July 4, 2020, https://www.gub.uy/system/documents/attachments/000/000/001/original/abc45c0eb835393 


\section{Agradecimientos}

Este paper es parte del proyecto "Investigación de campo y documental sobre autoridades de protección de datos en América", desarrollado con recursos de la Fundación de Apoyo a la Investigación del Estado de São Paulo (FAPESP) (https://bv.fapesp.br/pt/auxilios/105576/pesquisa-documental-e-de-campo-sobre-autoridadesde-protección-de-datos-en-américa-latina-el-concepto). Los autores agradecen a los revisores anónimos, cuyas críticas y recomendaciones fueron fundamentales para mejorar el texto original. Sin embargo, cualquier error o problema que sobrevenga son responsabilidad exclusiva de los autores. Asimismo, los autores agradecen a Carolina Ferreira de Medeiros por la revisión textual del manuscrito original.

\section{Biografía de los autores}

Alexandre Veronese, Ph.D. in Sociology by the Institute of Social and Political of the University of the State of Rio de Janeiro, Associate Professor at the University of Brasilia, Researcher at the Centre of Studies in European Union Law of the University do Minho (Portugal) and the Centre of Policies, Law, Economy and Communication Technologies (CCOM UnB), Coordinator of the Group of Telecommunications Law (GETEL UnB). E-mail: veronese@ccom.unb.br.

Amanda Nunes Lopes Espiñeira Lemos, Ph.D. candidate and Master in Law by the University of Brasília. Fellowship holder of Fiocruz (Brasília). Ex Junior Fellowship at Centro de Estudios de Telecomunicaciones de América Latina (cet.la). Researcher at the Group of Telecommunications Law (GETEL-UnB) and at Laboratory of Public Policy and Internet (LAPIN). E-mail: amandaespineira@gmail.com. 